\title{
Amiloride-blockable acid-sensing ion channels are leading acid sensors expressed in human nociceptors
}

\author{
Shinya Ugawa, Takashi Ueda, Yusuke Ishida, Makoto Nishigaki, Yasuhiro Shibata, \\ and Shoichi Shimada \\ Department of Anatomy II, Nagoya City University Medical School, Nagoya, Japan
}

\begin{abstract}
Many painful inflammatory and ischemic conditions such as rheumatoid arthritis, cardiac ischemia, and exhausted skeletal muscles are accompanied by local tissue acidosis. In such acidotic states, extracellular protons provoke the pain by opening cation channels in nociceptors. It is generally believed that a vanilloid receptor subtype-1 (VR1) and an acid-sensing ion channel (ASIC) mediate the greater part of acid-induced nociception in mammals. Here we provide evidence for the involvement of both channels in acid-evoked pain in humans and show their relative contributions to the nociception. In our psychophysical experiments, direct infusion of acidic solutions $(\mathrm{pH} \geq 6.0)$ into human skin caused localized pain, which was blocked by amiloride, an inhibitor of ASICs, but not by capsazepine, an inhibitor of VR1. Under more severe acidification ( $\mathrm{pH}$ 5.0) amiloride was less effective in reducing acid-evoked pain. In addition, capsazepine had a partial blocking effect under these conditions. Amiloride itself neither blocked capsaicin-evoked localized pain in human skin nor inhibited proton-induced currents in VR1-expressing Xenopus oocytes. Our results suggest that ASICs are leading acid sensors in human nociceptors and that VR1 participates in the nociception mainly under extremely acidic conditions.
\end{abstract}

J. Clin. Invest. 110:1185-1190 (2002). doi:10.1172/JCI200215709.

\section{Introduction}

Protons can modulate the activity of a number of receptors and ion channels expressed in nociceptors (1). Among such entities, they can directly activate vanilloid receptor subtype-1 (VR1) and acid-sensing ion channel ASICs $(2,3)$. VR1 is selectively expressed in polymodal nociceptors, which are responsive to noxiously thermal, mechanical, and chemical stimuli, and is broadly regarded as a major detector of multiple pain-producing stimuli. The contribution of VR1 to the $\mathrm{pH}$ sensitivity of nociceptors has been established in vitro by gene knockout experiments (4). However, the activation of the VR1 channel requires extremely severe acidification to $\mathrm{pH}$ less than $6.0(4,5)$, raising the possibility that another signal sensor that is more sensitive to protons than VR1 may be present in nociceptors, because, for example, skin nociceptors have activation thresholds as high as $\mathrm{pH} 6.9$ (6). In muscle and cardiac ischemia, an extracellular $\mathrm{pH}$ drop from 7.4 to 7.0 is sufficient to induce persistent activation of a subset of nociceptors (7-9).

Recent electrophysiological experiments have strongly suggested the involvement of ASICs (amiloride-blockable

Received for publication April 16, 2002, and accepted in revised form August 20, 2002.

Address correspondence to: Shinya Ugawa, Department of Anatomy II, Nagoya City University Medical School, 1 Kawasumi, Mizuho-cho, Mizuho-ku, Nagoya 467-8601, Japan.

Phone: 81-52-853-8126; Fax: 81-52-852-8887;

E-mail: ugawa@med.nagoya-cu.ac.jp.

Conflict of interest: No conflict of interest has been declared.

Nonstandard abbreviations used: vanilloid receptor subtype- 1

(VR1); acid-sensing ion channel (ASIC); dorsal root ganglion (DRG). proton-gated channel subunits expressed in mammalian central and peripheral nervous systems) (10) in nociception linked to acidoses. Sensory neurons from mice lacking ASIC-3 (nomenclature as in ref. 3 ) are severely deficient in their responses to acidic stimuli in vitro (11). The heterologously expressed ASIC-2b/ASIC-3 channel generates a biphasic inward current that is similar to the native proton-activated current in dorsal root ganglion (DRG) neurons (12). The ASIC- 3 channel is capable of reproducing the features of acid-evoked currents in cardiac nociceptors (13). Despite these observations, there is still controversy about the functional roles of ASICs in mammals, because proton detection through ASICs has not yet been demonstrated in vivo.

In this report, we evaluated the efficacy of amiloride (an inhibitor of ASICs) and capsazepine (an inhibitor of VR1) on acid-evoked pain in humans using a psychophysical method. To confirm the specificities of both drugs, we investigated their effects on capsaicinevoked pain using a similar psychophysical approach. Our results indicate the involvement of ASICs and VR1 in proton-induced pain in humans and show their relative importance in the nociception.

\section{Methods}

Psychophysical experiments. The following experiments were approved by the Ethics Committee of the Nagoya City University Medical School and conducted in accordance with the Declaration of Helsinki. A total of 56 healthy men, 21-41 years of age, participated in the study. All subjects stated that they had not used drugs of any kind within one week preceding the experiments. 
We determined $\mathrm{pH}$ dependency of acid-evoked pain at the beginning of the experiment. PBS $(0.01 \mathrm{M})$ was used as a basic infusion solution. The $\mathrm{pH}$ value of each stimulus solution was adjusted with $\mathrm{HCl}$. First, to establish a standard against which one could represent changes in pain, a pH 5.0 solution was infused hypodermically into the palmar side of the upper forearm at a flow rate of approximately $0.02 \mathrm{ml} / \mathrm{s}$ for 5 seconds, using a sterile syringe with a 29-gauge needle. After the infusion, the needle was pulled out from the skin. Next, eight kinds of solutions ( $\mathrm{pH} 7.4,7.3,7.2,7.0,6.5$, $6.0,5.5$, or 5.0) were applied randomly into the neighboring area of the ipsilateral forearm in the same manner, provided that injection sites were situated at least $3 \mathrm{~cm}$ from each other to avoid any kind of influence by previous infusions. The injections were performed at intervals of several minutes, during which the localized pain completely disappeared. Just after each application, the subject was asked to estimate the intensity of induced pain using a unipolar scale with zero (no pain) at one end and ten ( $\mathrm{pH}$ 5.0-evoked pain) at the other end. Any type of pain and/or unpleasant sensations present before or after the infusion was not considered in the estimation.

To assess the in vivo effects of amiloride or capsazepine on acid-evoked ( $\mathrm{pH}$ 6.0) pain, a unipolar scale with zero (no pain) at one end and ten ( $\mathrm{pH}$ 6.0-evoked pain) at the other end was used. After the standard $\mathrm{pH}$ 6.0 solution was injected, four kinds of solutions $(\mathrm{pH}$ $6.0 / 7.4$ solutions and the same solutions containing $200 \mu \mathrm{M}$ amiloride or $100 \mu \mathrm{M}$ capsazepine) were randomly applied to each subject in the same manner. We also investigated the analgesic effects of each drug on $\mathrm{pH}$ 7.0/6.5-evoked or 5.0-evoked pain using a unipolar scale with zero (no pain) at one end and ten $(\mathrm{pH} 6.5$ evoked and 5.0-evoked pain, respectively) at the other end. To examine the in vivo effects of amiloride or capsazepine on capsaicin-evoked pain, a unipolar scale with zero (no pain) at one end and ten $(20 \mu \mathrm{M}$ capsaicin; pH 7.4-evoked pain) at the other end was used instead. All studies were double blind and performed at room temperature. Data were analyzed using the Wilcoxon signed rank test. A prior level of significance was set at a $P$ value less than 0.05 .

\section{Results}

Dose-response relationship between proton concentration and magnitude of acid-evoked pain. We investigated relationships between $\mathrm{pH}$ values of acidic stimuli and intensity of proton-induced pain in human subjects $(n=8)$. The $\mathrm{pH} 5.0$ phosphate-buffered solution was first applied hypodermically into an intact skin area. In and around the injection site, the application produced a localized "pure" pain, which was sustained as long as a constant flow was maintained and rapidly disappeared when the flow was stopped. This consequence was in good agreement with observations described previously (14). We allotted ten points to this pain and established a unipolar scale for the following estimation.
Next, we randomly applied PBS solutions of various $\mathrm{pH}$ values to each subject in the same manner and asked subjects to estimate the evoked pain. In this pain model, the subjects started to feel pain at approximately $\mathrm{pH}$ 7.2, and the degree of associated pain increased with lowering the $\mathrm{pH}$ of the stimulus solutions, seeming to reach its saturation value at $\mathrm{pH}$ of approximately 5.5 (Figure 1a). The $\mathrm{pH} 7.4$ application elicited almost no painful sensation, indicating that intradermal pressure due to the injection itself evoked hardly any mechanical pain in the subjects tested. In rat skin, activities of a subpopulation of primary nociceptive afferents increase with lowering extracellular $\mathrm{pH}$ down to 5.2, but further acidification ( $\mathrm{pH}$ values lower than 5.2) elicited smaller nociceptor responses (6). Our data are in good accordance with this previous observation.
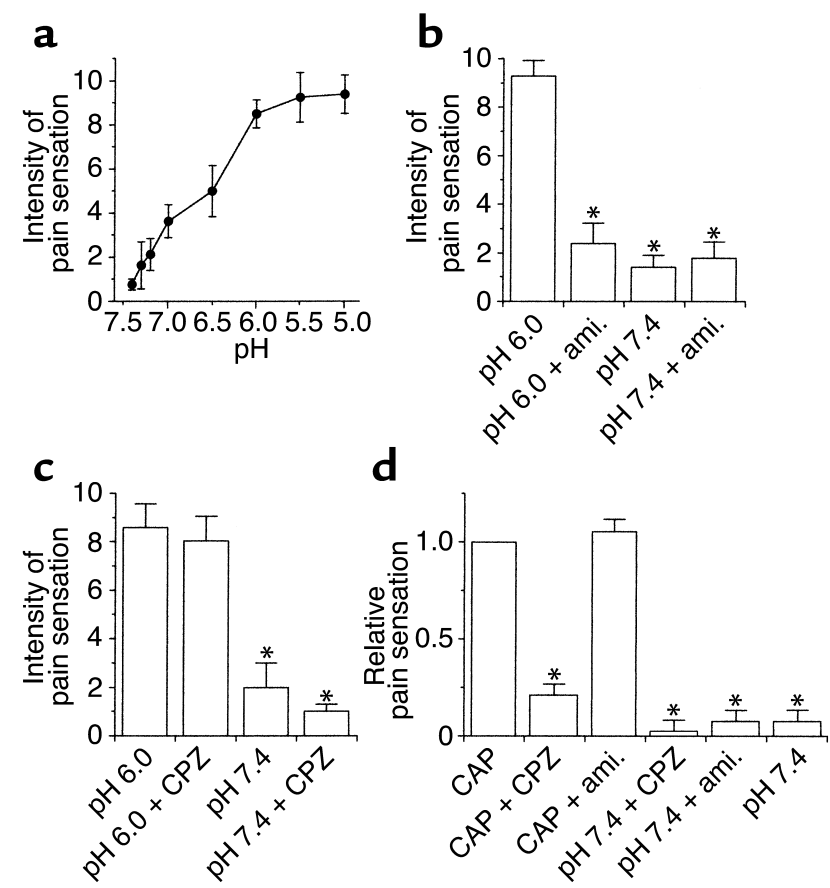

\section{Figure 1}

Pharmacological analyses of acid-evoked pain in humans. Mean $\pm \mathrm{SE}$ are shown for each data point. (a) Acids injected hypodermically into human skin provoke pain in a $\mathrm{pH}$-dependent manner $(n=8)$. The subjects tested started to feel pain at approximately $\mathrm{pH} 7.2$, and the degree of the evoked painful sensation was well correlated with the magnitude of acidification, with a saturation value at approximately $\mathrm{pH}$ 5.5. (b) Instant effects of an ASIC blocker, amiloride (ami.; 200 $\mu \mathrm{M})(n=10)$. Amiloride treatment potently blocked acid-evoked $(\mathrm{pH}$ 6.0) pain. The vertical axis shows estimated pain values $\left({ }^{*} P<0.01\right.$ vs. second $\mathrm{pH} 6.0$ treatments). (c) Effects of a VR1 blocker, capsazepine $(\mathrm{CPZ} ; 100 \mu \mathrm{M})$, on acid-evoked $(\mathrm{pH} 6.0)$ pain $\left({ }^{*} P<0.01\right.$ vs. second $\mathrm{pH} 6.0$ treatments) $(n=10)$. Simultaneous applications of capsazepine did not display any analgesic effects on the $\mathrm{pH} 6.0$ stimuli. The capsazepine applications $(\mathrm{pH} 7.4)$ themselves provoked no significant pain. (d) In vivo effects of capsazepine $(100 \mu \mathrm{M})$ and amiloride $(200 \mu \mathrm{M})$ on $20 \mu \mathrm{M}$ capsaicin-evoked (CAP; $\mathrm{pH}$ 7.4) pain in human subjects. Capsazepine potently suppressed the capsaicinevoked pain in the subjects tested, whereas the amiloride induced no analgesic effects $(n=8)$. Data are plotted relative to the capsaicinevoked pain ( $P<0.05$ vs. capsaicin treatments). 
Amiloride potently blocks proton-induced pain in bumans. Before injecting amiloride and capsazepine under human skin, we checked the specificities of both drugs by oocyte electrophysiology as follows: $100 \mu \mathrm{M}$ capsazepine and $200 \mu \mathrm{M}$ amiloride had no influence on acid-evoked ( $\mathrm{pH}$ 6.0 for ASICs and $\mathrm{pH} 5.4$ for VR1) currents of ASIC-expressing (ASIC-1a, ASIC-1b, ASIC-2a, or ASIC-3) and VR1-expressing oocytes, respectively; the capsazepine application potently blocked the VR1 channel activity; the amiloride treatment was highly effective in reducing the currents of the ASIC-1a, ASIC-1b, ASIC-2a, and ASIC-3 channels (data not shown). We used rat orthologues for the functional expression in Xenopus oocytes because rat ASIC and VR1 channels have no significant differences in affinities for amiloride and capsazepine from their human counterparts $(5,12,15-25)$. The experiments were not performed on the ASIC- $2 b$ and ASIC- 4 channels because they are inactive by themselves $(12,26-28)$.

We then investigated the in vivo effects of amiloride, the sensitivity of which is a common notable characteristic of ASICs, on the acid-evoked pain in ten volunteers (Figure 1b). When $\mathrm{pH} 6.0$ solutions were applied for the second time, most of the subjects reported almost the same magnitude of pain as the first time. In contrast, the $\mathrm{pH} 7.4$ solutions elicited much less pain than the $\mathrm{pH} 6.0$ treatments, indicating that the subjects were able to properly discriminate between the two kinds of stimuli $(P=0.0051)$. The most striking observation was that simultaneous applications of $200 \mu \mathrm{M}$ amiloride reduced the acid-evoked pain almost to the $\mathrm{pH} 7.4$ control level $(P=0.0077)$, implying that ASICs expressed in nociceptors are likely to play important roles in detecting noxiously acidic stimuli.

We examined the effects of $100 \mu \mathrm{M}$ capsazepine in another ten volunteers and found that simultaneous applications of capsazepine did not have any analgesic effects on the pH 6.0 stimuli $(P=0.7989)$ (Figure 1c). This concentration of capsazepine is high enough for the complete inhibition of proton- and capsaicin-activated currents of human VR1 (5), and capsazepine applications ( $\mathrm{pH}$ 7.4) themselves provoked no significant pain in the subjects. We also confirmed that 100 $\mu \mathrm{M}$ capsazepine potently suppressed $20 \mu \mathrm{M}$ capsaicinevoked ( $\mathrm{pH}$ 7.4) pain in other human subjects $(n=8)$ (Figure 1d). These results strongly suggest that VR1 makes little contribution to acid-evoked pain at $\mathrm{pH}$ greater than or equal to 6.0.

The possibility that the amiloride application affected components of the pain transduction machinery besides ASICs was clearly excluded (Figure 1d). The subjects tested felt almost the same magnitude of capsaicin-evoked pain even in the presence of $200 \mu \mathrm{M}$ amiloride when compared with that in the absence of the drug $(P=0.0117)$. These results indicate the soundness of the pain-conducting pathways themselves even under the amiloride treatments. In other words, the analgesic effects of amiloride were restricted to the initial phase of the proton-induced responses in the noci- ceptors. Taken together, the above psychophysical data strongly suggest that amiloride relieved the acidevoked pain through its specific blocking effects on ASICs in the nociceptors.

Effects of amiloride and capsazepine on acid-evoked pain at various $p H$ stages. We further examined the analgesic effects of amiloride on acid-evoked pain at a higher $\mathrm{pH}$ range than 6.0. A pH 6.5 solution was used to establish a standard, and five kinds of PBS solutions (indicated in Figure 2a) were randomly applied $(n=10)$. It was confirmed that pain provoked by the second $\mathrm{pH} 6.5$ application showed almost the same magnitude as that evoked by the first $\mathrm{pH} 6.5$ application in each subject. The intensity of the induced pain was attenuated in a $\mathrm{pH}$-dependent manner with lowering proton concentration. Even at these $\mathrm{pH}$ stages, $200 \mu \mathrm{M}$ amiloride potently suppressed the acid-evoked pain down to the $\mathrm{pH} 7.4$ control level $(P=0.0051$ and 0.0069 at $\mathrm{pH} 6.5$ and 7.0, respectively). These findings strongly suggest that ASICs located in human nociceptors chiefly function as acid sensors at $\mathrm{pH}$ greater than 6.0.

We then investigated effects of amiloride and capsazepine at a lower $\mathrm{pH}$ range than $6.0(n=10)$. We selected a pH 5.0 solution as a standard stimulus, because both ASICs and VR1 are likely to be active under this acidic condition (5). Interestingly, the analgesic effects of amiloride at $\mathrm{pH} 5.0$ were less potent than those observed at $\mathrm{pH}$ greater than or equal to $6.0(P=0.0077)$, while $100 \mu \mathrm{M}$ capsazepine displayed partial blocking effects on the proton-induced pain $(P=0.0117)$ (Figure $2 \mathrm{~b})$.
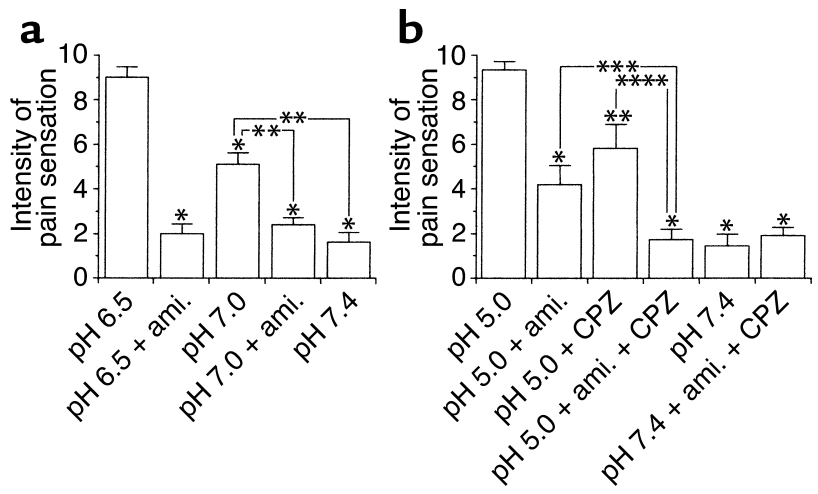

\section{Figure 2}

Analgesic effects of amiloride (ami.; $200 \mu \mathrm{M}$ ) and capsazepine (CPZ; $100 \mu \mathrm{M}$ ) on acid-evoked pain in human subjects at various $\mathrm{pH}$ stages. Mean \pm SE are shown for each data point. (a) Effects of amiloride on $\mathrm{pH} 6.5$ - and 7.0-induced pain $(n=10)$. The intensity of provoked painful sensation was attenuated in a $\mathrm{pH}$-dependent manner with lowering proton concentration. Amiloride treatment potently suppressed the acid-evoked pain down to the $\mathrm{pH} 7.4$ control level $\left({ }^{*} P<0.01\right.$ vs. second $\mathrm{pH} 6.5$ treatments; ${ }^{*} P<0.01$ vs. $\mathrm{pH}$ 7.0 treatments). (b) Effects of the amiloride and capsazepine on $\mathrm{pH}$ 5.0 -induced pain $(n=10)$. The analgesic effects of the amiloride at $\mathrm{pH} 5.0$ were less potent than those observed at $\mathrm{pH}$ greater than or equal to 6.0, while the capsazepine displayed partial blocking effects on the pain instead $\left({ }^{*} P<0.01\right.$ and ${ }^{*} P<0.05$ vs. second $\mathrm{pH} 5.0$ treatments). Simultaneous applications of both drugs almost completely abolished the pain $\left({ }^{* *} P=0.0117\right.$, and $\left.{ }^{* * * *} P=0.0173\right)$. 
Simultaneous applications of both drugs almost completely relieved the pain $(P=0.0117$ and $P=0.0173$; see Figure $2 \mathrm{~b}$ ). These findings indicated that human VR1 participated in the proton reception to provoke the pain at this $\mathrm{pH}$ stage. Taken together with our electrophysiological data, we concluded that ASICs were leading acid sensors to detect noxious acidification in the human body and that VR1 contributed to the nociception mainly under extremely acidic conditions.

\section{Discussion}

Using the psychophysical method, we demonstrated here that ASICs (and not VR1) function as acid sensors in vivo within a pathophysiologically relevant $\mathrm{pH}$ range $(\mathrm{pH} \geq 6.0)$ and that a diuretic agent, amiloride, may be useful as an analgesic against proton-induced localized pain in the $\mathrm{pH}$ range of 7.2-6.0. The human subjects tested felt pain even at $\mathrm{pH} 7.0$, which is low enough for the activation of the ASIC- $1 \mathrm{a}$ and ASIC- 3 channels (20, 24). In contrast, human VR1 cannot start to open until extracellular $\mathrm{pH}$ drops to 6.0 or less (5), implying that the ASIC channels show an order of magnitude with a higher affinity for protons than the VR1. (As described elsewhere, the rat ASIC- $1 \mathrm{~b}$ channel starts to open at $\mathrm{pH}$ 6.5 , and the rat and human ASIC-2a channels do so at approximately $\mathrm{pH} 6.0$; refs. 18, 26, 29) This lower activation threshold of the VR1 channel raises the possibility that VR1 may contribute to acid-evoked pain mainly under extremely acidic conditions $(\mathrm{pH} \leq 6.0)$ and that VR1 indeed did so in our pain model (see Figure $2 \mathrm{~b}$ ). For this reason, more attention should be given to the ASIC family of cation channels and to the relative importance of ASICs and VR1 in the nociception.

It is well known that protons increase the potency of heat as a VR1 agonist by lowering the threshold for channel activation by the stimulus (17). Extracellular acidification to $\mathrm{pH} 6.4$ is not sufficient to activate the rat VR1 channel at room temperature, whereas a considerable amount of the channel current is constantly observed at the same $\mathrm{pH}$ at body temperature (4). These findings indicate that a decrease in extracellular $\mathrm{pH}$ to 6.4 is low enough to activate the channel under normal physiological conditions. Despite the above fact, however, high concentrations of capsazepine $(100 \mu \mathrm{M})$ showed no analgesic effect on $\mathrm{pH}$ 6.0 -induced pain in the human skin (see Figure 1c). Although the acidification to $\mathrm{pH} 6.0$ probably activates the human VR1 channel in the skin nociceptors, the $\mathrm{pH}$ level may not be low enough for VR1 to cause the membrane potential to reach the firing level of the peripheral nociceptive afferents.

There are several reports on the effects of amiloride in proton-induced nociception or irritation. Pretreatment with amiloride significantly reduced irritant sensations in human subjects, which were elicited by applications of citric acid on the dorsal surface of the tongue, but did not reduce capsaicin-evoked oral irritation, indicating the potential involvement of an amiloride-sensitive mechanism in acid-induced irrita- tion (30). These observations may provide support for our idea that ASICs are the main acid sensors in human nociceptors, although there is a gap in experimental systems between the irritation test and our pain model. On the other hand, it was shown previously that amiloride enhanced acid-evoked responses of rat $\mathrm{C}$ fibers in the presence of nonphysiologically high concentrations of $\mathrm{Na}^{+}(31)$. The authors speculated that amiloride blocked a Na$/ \mathrm{H}^{+}$exchanger in the terminal endings of the nociceptive neurons (all isoforms of $\mathrm{Na}^{+} / \mathrm{H}^{+}$antiporters are known to be sensitive to high concentrations of amiloride), preventing extrusion of permeating protons, then the incremental intracellular protons prolonged the $\mathrm{pH}$ responses. Their experiments were performed using high concentrations of amiloride in the presence of $217 \mathrm{mM}$ $\mathrm{Na}^{+}$to enhance its effects on $\mathrm{Na}^{+} / \mathrm{H}^{+}$antiporters. Their application period of amiloride was 5 minutes, and the peak responses of the $\mathrm{C}$ fibers were observed two to 10 minutes after the treatment with amiloride. In contrast, our psychophysical experiments were designed to detect the effect of amiloride on ASICs. The acidification periods and/or application periods of amiloride were 5 seconds in the studies. The latency of the pain responses were very short (within a second), and the analgesic effect of the amiloride occurred simultaneously with the evoked painful sensation. The effects of amiloride have also been tested in other animal models. In the rat nervous system, inhibition of $\mathrm{Na}^{+} / \mathrm{H}^{+}$ exchangers in the GABAergic neurons by amiloride is postulated to be the mechanism by which the drug prevents $G_{A B A}$ receptor-mediated pentobarbitalinduced facilitation of nocifensive reflexes (32). Systemic, spinal, and supraspinal administration of amiloride caused dose-related antinociception against acetic acid-induced abdominal constriction in the mouse, but the effects may be attributable to the broad nonspecific actions of amiloride as described (33).

To date, four ASIC genes - ASIC-1 (ASIC-1a and its splice variant ASIC-1b), ASIC-2 (the two splice variants ASIC-2a and ASIC-2b), ASIC-3, and ASIC-4 - have been identified in mammalian organisms (3). Which ASIC is located in nociceptors? According to previous in situ hybridization experiments, ASIC-1a, ASIC-1b, ASIC-2b, and ASIC-3 are the main molecular entities present in rat DRG neurons $(12,15,16,18,27,28,34$, 35 ), and, furthermore, protein expressions for the ASICs are identified in many of the SP- or IB4-positive neurons that correspond to neuropeptidergic or nonpeptidergic unmyelinated nociceptors $(36,37)$. In humans, expressions of ASIC-1a, ASIC- $2 b$, and ASIC-3 in the DRG neurons have been confirmed by previous immunoblot analyses (38). Their immunoreactivities are more or less found in the small-to-medium-diame$\operatorname{ter}(<50 \mu \mathrm{m}$ in somata diameter) sensory neurons, indicating that the human ASIC clones probably serve as acid sensors in nociceptive neurons, because small- and medium-diameter sensory neurons give rise to most of nociceptors $(1,2)$. Interestingly, human ASIC-3 is more 
widely distributed than its murine counterpart, even in the non-neuronal tissues $(25,38)$, so the ASIC-3 may play a more critical role in the broad range of nociception. Expressions of ASIC-1b, ASIC-2a, and ASIC-4 in human sensory neurons remain to be elucidated.

In acidotic states, extracellular protons elicit both transient and sustained excitatory responses in sensory neurons, the latter of which may account for persistent pain in patients (39). In contrast to the ASIC-1a and ASIC-1b channels, which generate transient currents in response to acidic stimuli, the ASIC-3 channel is capable of generating biphasic inward currents that contain the sustained component in both animal and human species $(15,16,18-21,24,25)$. According to recent reports, ASIC-3 is largely colocalized with ASIC-2b in single sensory neurons to form a heteromeric complex in rat and mouse DRG $(40,41)$, while the heteromer also produces a biphasic current with a similar steadystate component. Thus, it is possible that the ongoing pain (the magnitude of which was constant as long as the flow rate was properly maintained) reported by the subjects tested at $\mathrm{pH}$ less than or equal to 6.0 was due to the long-lasting currents originated from the ASIC-3 subunit in the nociceptors. Unlike heterologous expression systems, it was difficult to cause a rapid $\mathrm{pH}$ drop in human skin. Therefore, another possibility is that the extracellular proton concentrations around the skin nociceptors were gradually decreased by the acid injection, and then the ASIC-1a or ASIC-1b channel started to open with slower kinetics than those observed in vitro, leading to the persistent pain. This proposal is supported by the finding that the sustained current of the ASIC-3 channel is not inhibited by amiloride $(16,24$, $25)$, whereas the subjects recognized almost no pain in the presence of the drug. Needless to say, sustained VR1 currents are sure to contribute to the generation of the persistent pain under more severe acidification.

The mechanisms of regulation of ASICs and VR1 by inflammatory mediators have been described previously in several reports. In a Freund's adjuvant-induced inflammation model, ASIC-1a transcripts appeared in some larger $A \beta$ fibers, which have no role in nociception under physiologically normal conditions (36). The mRNA levels of ASIC-1a, ASIC-2b, and ASIC-3 in the DRG were highly increased 1-2 days after the adjuvant application, whereas the VR1 transcript level was relatively stable. Intriguingly, nonsteroidal anti-inflammatory drugs prevented the increase in ASIC expression, and some of them directly inhibited ASIC-type membrane currents in sensory neurons. ASIC-3 (but not ASIC-1 or ASIC-2) protein expression in human enteric neurons was increased in the inflamed Crohn disease intestine, although the functional significance of the expression of the ASIC-1, -2 , and -3 by the neurons is unknown (38). Proalgesic agents, bradykinin and nerve growth factor, are able to sensitize VR1 at sites of injury and inflammation, which provides a possible explanation for both mediated potentiation of thermal sensitivity in vivo (42). Further studies are needed to eluci- date the reciprocal relationships between ASICs and VR1 at various pathophysiological sites.

In summary, ASICs are leading acid sensors in human nociceptors, contributing to acid-induced nociception within a pathophysiologically relevant $\mathrm{pH}$ range $(\mathrm{pH} \geq 6.0)$. VR1 participates in proton detection mainly under more acidic conditions $(\mathrm{pH} \leq 6.0)$. Future investigations on pain associated with local tissue acidoses in various pathological conditions should give consideration to the relative contributions of ASICs and VR1 to the nociception.

\section{Acknowledgments}

The authors thank Koichi Noguchi and Tetsuo Fukuoka for helpful discussions and Katsuyuki Tanaka and Kenji Kajita for skillful technical assistance. This work was supported by a research grant to $S$. Ugawa from the Japan Society for the Promotion of Science.

1. Kress, M., and Reeh, P.W. 1996. Chemical excitation and sensitization in nociceptors. In Neurobiology of nociceptors. F. Cervero and C. Belmonte, editors. Oxford University Press. New York, New York, USA. 258-297.

2. Julius, D., and Basbaum, A.I. 2001. Molecular mechanisms of nociception. Nature. 413:203-210.

3. Reeh, P.W., and Kress, M. 2001. Molecular physiology of proton transduction in nociceptors. Curr. Opin. Pharmacol. 1:45-51.

4. Caterina, M.J., and Julius, D. 2001. The vanilloid receptor: a molecular gateway to the pain pathway. Annu. Rev. Neurosci. 24:487-517.

5. Cortright, D.N., et al. 2001. The tissue distribution and functional characterization of human VR1. Biochem. Biophys. Res. Commun. 281:1183-1189.

6. Steen, K.H., Reeh, P.W., Anton, F., and Handwerker, H.O. 1992. Protons selectively induce lasting excitation and sensitization to mechanical stimulation of nociceptors in rat skin, in vitro. J. Neurosci. 12:86-95.

7. Issberner, U., Reeh, P.W., and Steen, K.H. 1996. Pain due to tissue acidosis: a mechanism for inflammatory and ischemic myalgia? Neurosci. Lett. 208:191-194.

8. Cobbe, S.M., and Poole-Wilson, P.A. 1980. The time of onset and severity of acidosis in myocardial ischaemia. J. Mol. Cell Cardiol. 12:745-760.

9. Cobbe, S.M., and Poole-Wilson, P.A. 1982. Continuous coronary sinus and arterial $\mathrm{pH}$ monitoring during pacing-induced ischaemia in coronary artery disease. Br. Heart J. 47:369-374.

10. Kellenberger, S., and Schild, L. 2002. Epithelial sodium channel/degenerin family of ion channels: a variety of functions for a shared structure. Physiol. Rev. 82:735-767.

11. Price, M.P., et al. 2001. The DRASIC cation channel contributes to the detection of cutaneous touch and acid stimuli in mice. Neuron. 32:1071-1083.

12. Lingueglia, E., et al. 1997. A modulatory subunit of acid sensing ion channels in brain and dorsal root ganglion cells. J. Biol. Chem. 272:29778-29783.

13. Sutherland, S.P., Benson, C.J., Adelman, J.P., and McCleskey, E.W. 2001. Acid-sensing ion channel 3 matches the acid-gated current in cardiac ischemia-sensing neurons. Proc. Natl. Acad. Sci. USA. 98:711-716.

14. Steen, K.H., and Reeh, P.W. 1993. Sustained graded pain and hyperalgesia from harmless experimental tissue acidosis in human skin. Neurosci. Lett. 154:113-116.

15. Waldmann, R., Champigny, G., Bassilana, F., Heurteaux, C., and Lazdunski, M. 1997. A proton-gated cation channel involved in acid-sensing. Nature. 386:173-177.

16. Waldmann, R., et al. 1997. Molecular cloning of a non-inactivating proton-gated $\mathrm{Na}^{+}$channel specific for sensory neurons. J. Biol. Chem. 272:20975-20978.

17. Tominaga, M., et al. 1998. The cloned capsaicin receptor integrates multiple pain-producing stimuli. Neuron. 21:531-543.

18. Chen, C.C., England, S., Akopian, A.N., and Wood, J.N. 1998. A sensory neuron-specific, proton-gated ion channel. Proc. Natl. Acad. Sci. USA. 95:10240-10245.

19. Bässler, E.L., Ngo-Anh, T.J., Geisler, H.S., Ruppersberg, J.P., and Gründer, S. 2001. Molecular and functional characterization of acid-sensing ion channel (ASIC) 1b. J. Biol. Chem. 276:33782-33787.

20. Askwith, C.C., et al. 2000. Neuropeptide FF and FMRFamide potentiate acid-evoked currents from sensory neurons and proton-gated DEG/ENaC channels. Neuron. 26:133-141.

21. Berdiev, B.K., et al. 2001. pH alterations "reset" $\mathrm{Ca}^{2+}$ sensitivity of brain 
$\mathrm{Na}^{+}$channel 2, a degenerin/epithelial $\mathrm{Na}^{+}$ion channel, in planar lipid bilayers. J. Biol. Chem. 276:38755-38761.

22. García-Añoveros, J., Derfler, B., Neville-Golden, J., Hyman, B.T., and Corey, D.P. 1997. BNaC1 and $\mathrm{BNaC} 2$ constitute a new family of human neuronal sodium channels related to degenerins and epithelial sodium channels. Proc. Natl. Acad. Sci. USA. 94:1459-1464.

23. Adams, C.M., Snyder, P.M., Price, M.P., and Welsh, M.J. 1998. Protons activate brain $\mathrm{Na}^{+}$channel 1 by inducing a conformational change that exposes a residue associated with neurodegeneration. J. Biol. Chem. 273:30204-30207.

24. de Weille, J.R., Bassilana, F., Lazdunski, M., and Waldmann, R. 1998. Identification, functional expression and chromosomal localisation of a sustained human proton-gated cation channel. FEBS Lett. 433:257-260.

25. Babinski, K., Le, K.T., and Seguela, P. 1999. Molecular cloning and regional distribution of a human proton receptor subunit with biphasic functional properties. J. Neurochem. 72:51-57.

26. Ugawa, S., Ueda, T., Minami, Y., Horimoto, M., and Shimada, S. 2001. A single amino acid substitution in MDEG2 specifically alters desensitization of the proton-activated cation current. Neuroreport. 12:2141-2145.

27. Akopian, A.N., Chen, C.C., Ding, Y., Cesare, P., and Wood, J.N. 2000. A new member of the acid-sensing ion channel family. Neuroreport. 11:2217-2222.

28. Gründer, S., Geissler, H.S., Bässler, E.L., and Ruppersberg, J.P. 2000. A new member of acid-sensing ion channels from pituitary gland. Neuroreport. 11:1607-1611.

29. Adams, C.M., Snyder, P.M., Price, M.P., and Welsh, M.J. 1998. Protons activate brain $\mathrm{Na}^{+}$channel 1 by inducing a conformational change that exposes a residue associated with neurodegeneration. J. Biol. Chem. 273:30204-30207.

30. Dessirier, J.M., O’Mahony, M., Iodi-Carstens, M., and Carstens, E. 2000 Sensory properties of citric acid: psychophysical evidence for sensitization, self-desensitization, cross-desensitization and cross-stimulusinduced recovery following capsaicin. Chem. Senses. 25:769-780.
31. Steen, K.H., Wegner, H., and Reeh, P.W. 1999. The $\mathrm{pH}$ response of rat cutaneous nociceptors correlates with extracellular $\left[\mathrm{Na}^{+}\right]$and is increased under amiloride. Eur. J. Neurosci. 11:2783-2792.

32. Archer, D.P., and Roth, S.H. 1999. Acetazolamide and amiloride inhibit pentobarbital-induced facilitation of nocifensive reflexes. Anesthesiology. 90:1158-1164.

33. Ferreira, J., Santos, A.R., and Calixto, J.B. 1999. Antinociception produced by systemic, spinal and supraspinal administration of amiloride in mice. Life Sci. 65:1059-1066.

34. Waldmann, R., and Lazdunski, M. 1998. $\mathrm{H}^{+}$-gated cation channels: neuronal acid sensors in the NaC/DEG family of ion channels. Curr. Opin. Neurobiol. 8:418-424.

35. Price, M.P., et al. 2000. The mammalian sodium channel BNC1 is required for normal touch sensation. Nature. 407:1007-1011.

36. Voilley, N., de Weille, J., Mamet, J., and Lazdunski, M. 2001. Nonsteroid anti-inflammatory drugs inhibit both the activity and the inflammation-induced expression of acid-sensing ion channels in nociceptors. J. Neurosci. 15:8026-8033.

37. Snider, W.D., and McMahon, S.B. 1998. Tackling pain at the source: new ideas about nociceptors. Neuron. 20:629-632.

38. Yiangou, Y., et al. 2001. Increased acid-sensing ion channel ASIC-3 in inflamed human intestine. Eur. J. Gastroenterol. Hepatol. 13:891-896.

39. Bevan, S., and Geppetti, P. 1994. Protons: small stimulants of capsaicinsensitive sensory nerves. Trends. Neurosci. 17:509-512.

40. Alvarez de la Rosa, D., Zhang, P., Shao, D., White, F., and Canessa, C.M. 2002. Functional implications of the localization and activity of acidsensitive channels in rat peripheral nervous system. Proc. Natl. Acad. Sci. USA. 19:2326-2331.

41. Benson, C.J., et al. 2002. Heteromultimers of DEG/ENaC subunits form $\mathrm{H}^{+}$-gated channels in mouse sensory neurons. Proc. Natl. Acad. Sci. USA. 19:2338-2343.

42. Chuang, H.H., et al. 2001. Bradykinin and nerve growth factor release the capsaicin receptor from PtdIns $(4,5) \mathrm{P}_{2}$-mediated inhibition. Nature. 411:957-962. 